\title{
Correction to: FAM83B inhibits ovarian cancer cisplatin resistance through inhibiting Wnt pathway
}

Shanyang He@, Wei Wang, Zhiyong Wan, Hongwei Shen, Yunhe Zhao, Zeshan You, Jun Liu and Liwen Zhu

Correction to: Oncogenesis

https://doi.org/10.1038/s41389-020-00301-y

published online 9 January 2021

The original version of this article unfortunately contained a mistake. The following correction has therefore been made in the original: Affiliations 1 and 3 were interchanged and affiliation 2 was incomplete. The original article has been corrected.

Published online: 10 February 2021 